\title{
Plug-in Safe-by-Design Nanoinorganic Antibacterials
}

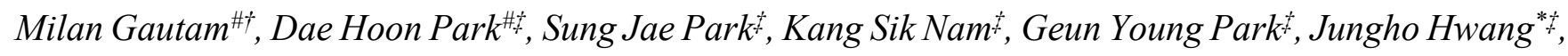
Chul Soon Yong ${ }^{\dagger}$, Jong Oh Kim ${ }^{* \dagger}$, Jeong Hoon Byeon ${ }^{*} \perp$

Mr. Milan Gautam, Dr. Chul SoonYong, and Dr. Jong Oh Kim

†College of Pharmacy, Yeungnam University, Gyeongsan 38541, Republic of Korea

Mr. Dae Hoon Park, Mr. Sung Jae Park, Mr. Kang Sik Nam, Mr. Geun Young Park, and Dr. Jungho Hwang

tSchool of Mechanical Engineering, Yonsei University, Seoul 03722, Republic of Korea

Dr. Jeong Hoon Byeon

${ }^{\perp}$ School of Mechanical Engineering, Yeungnam University, Gyeongsan 38541, Republic of Korea

\#These authors contributed equally to this work.

*Corresponding authors: hwangjh@yonsei.ac.kr, jongohkim@yu.ac.kr, and postjb@yu.ac.kr 

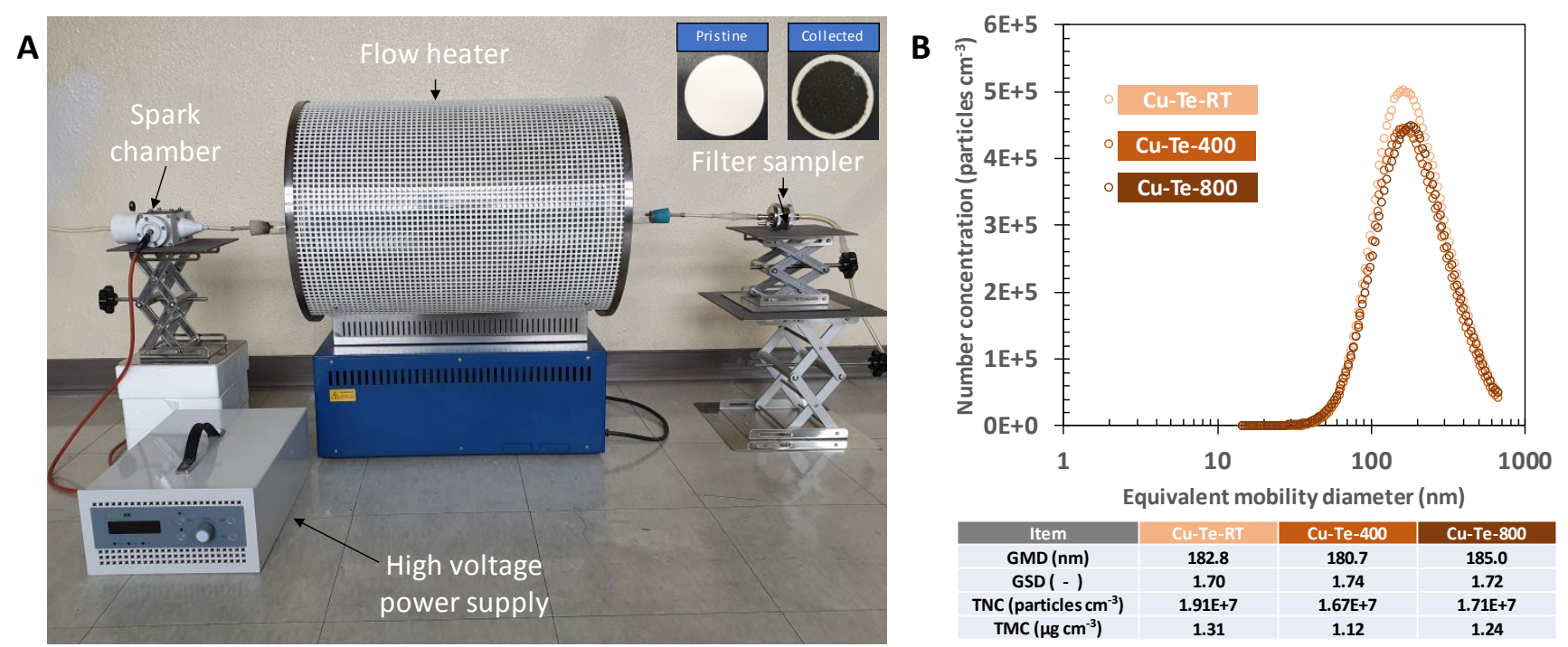

(A) Digital image of the plug-in system for manufacturing different $\mathrm{Cu}$-Te constructs; a serial connection of the spark chamber, flow heater, and filter sampler; the high voltage power supply was connected to the spark chamber for spark ablation while the filter sampler was installed right after the flow heater to directly collect the constructs (inset image for "Collected"). (B) Aerosol size distributions of Cu-Te NPs after passing through a flow heater operating at $\mathrm{RT}, 400^{\circ} \mathrm{C}$, or $800^{\circ} \mathrm{C}$; the summaries of size distributions (geometric mean diameter, geometric standard deviations, total number concentration, and total mass concentration) are provided in the bottom table. For SMPS measurements, the particle-laden flow was directly sampled immediately after the flow heater at $0.3 \mathrm{~L} \mathrm{~min}^{-1}$. 
FIGURE S2
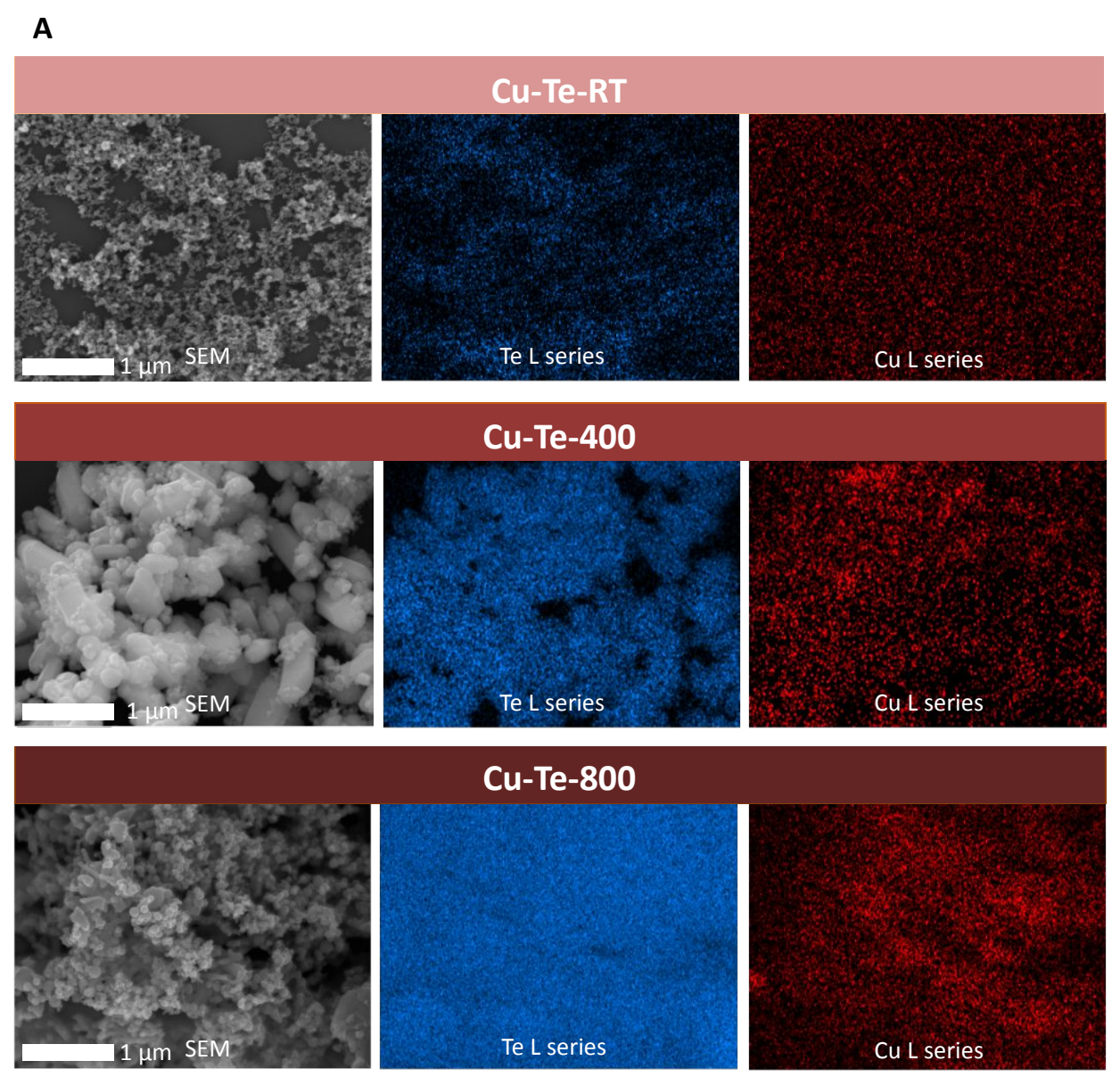

B

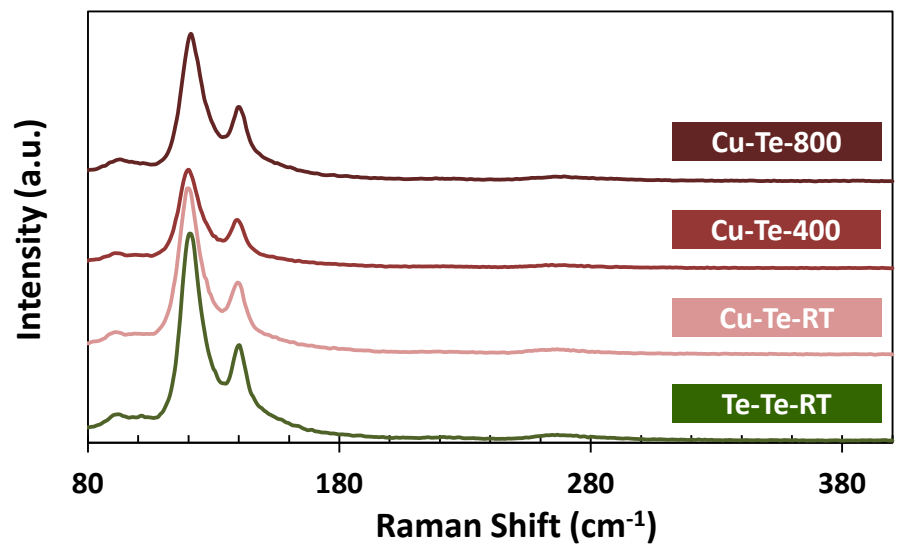

Elemental analysis of $\mathrm{Cu}-\mathrm{Te}$ constructs; (A) selected region (SEM image) for acquiring EDS (Cu and Te elements) maps of $\mathrm{Cu}-\mathrm{Te}$ constructs from the flow heater with operating temperatures; (B) Raman spectra of particles on ultraflat glass substrates. 
FIGURE S3
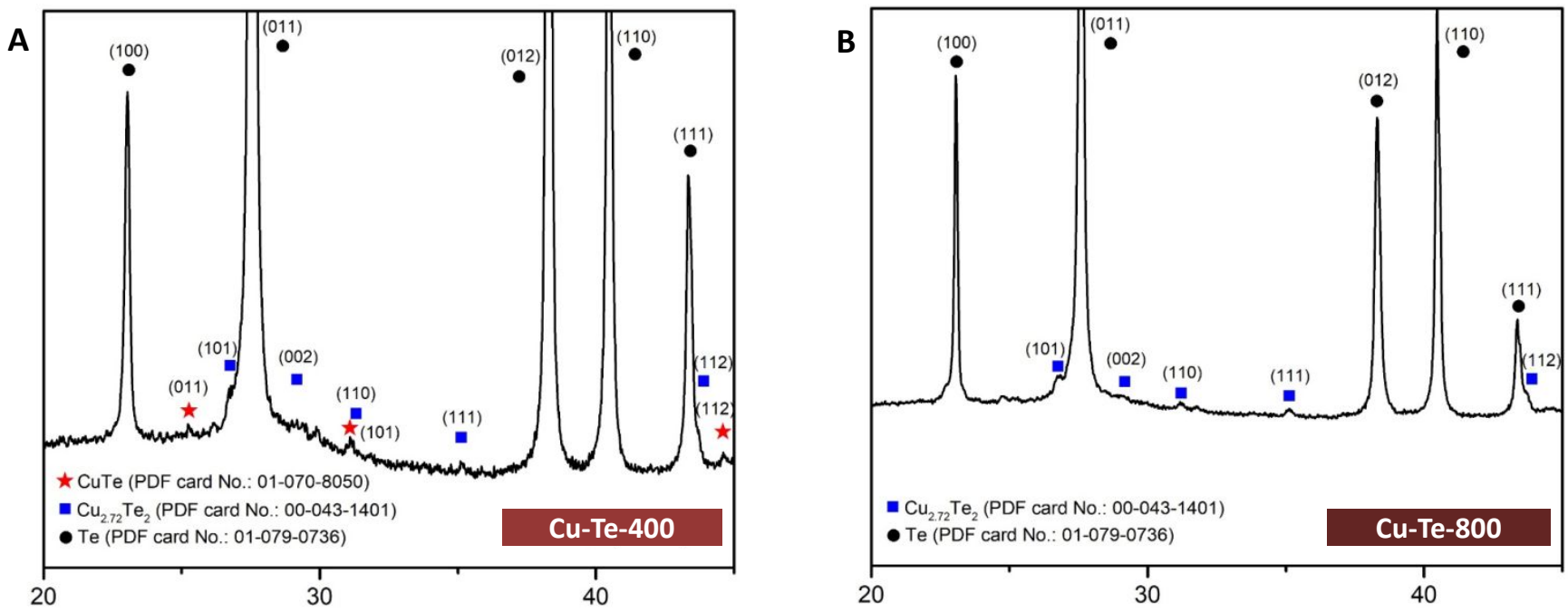

Enlarged XRD profiles of the (A) $\mathrm{Cu}-\mathrm{Te}-400$ and (B) $\mathrm{Cu}-\mathrm{Te}-800$ constructs shown in Figure 3; characteristic peaks of $\mathrm{Cu}-\mathrm{Te}$ crystals can be seen clearly in the particles, although Te peaks are significantly more intense than those of $\mathrm{Cu}-\mathrm{Te}$. 
FIGURE S4

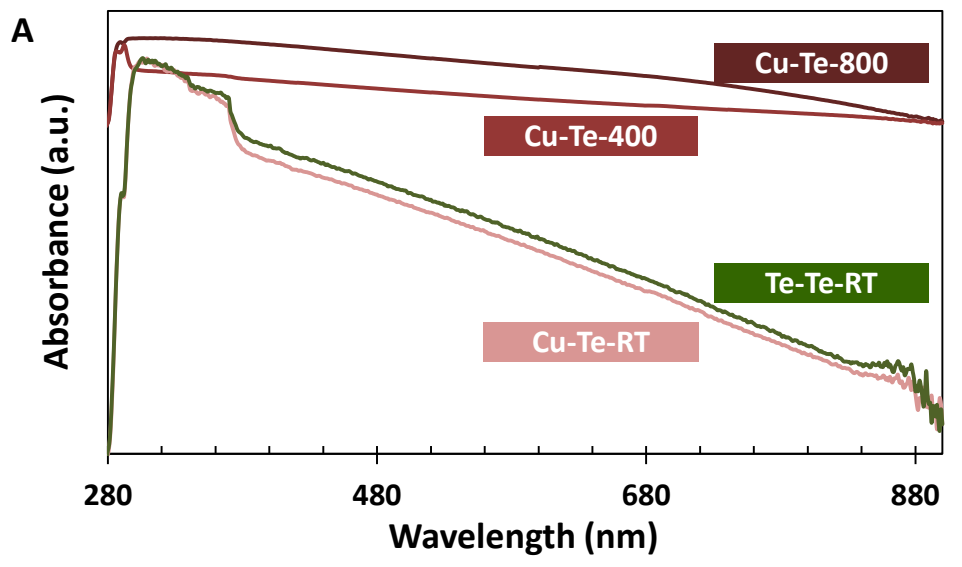

B

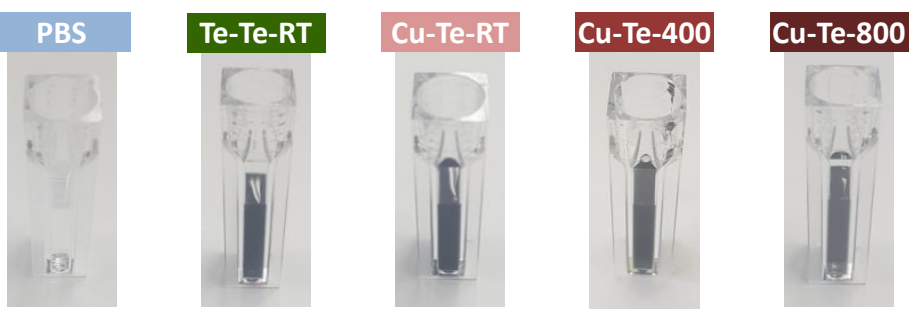

(A) Light absorption spectra of $\mathrm{Cu}-\mathrm{Te}$ constructs from the flow heater at different operating temperatures; (B) digital images of $\mathrm{Cu}-\mathrm{Te}$ particles dispersed in PBS. 
FIGURE S5
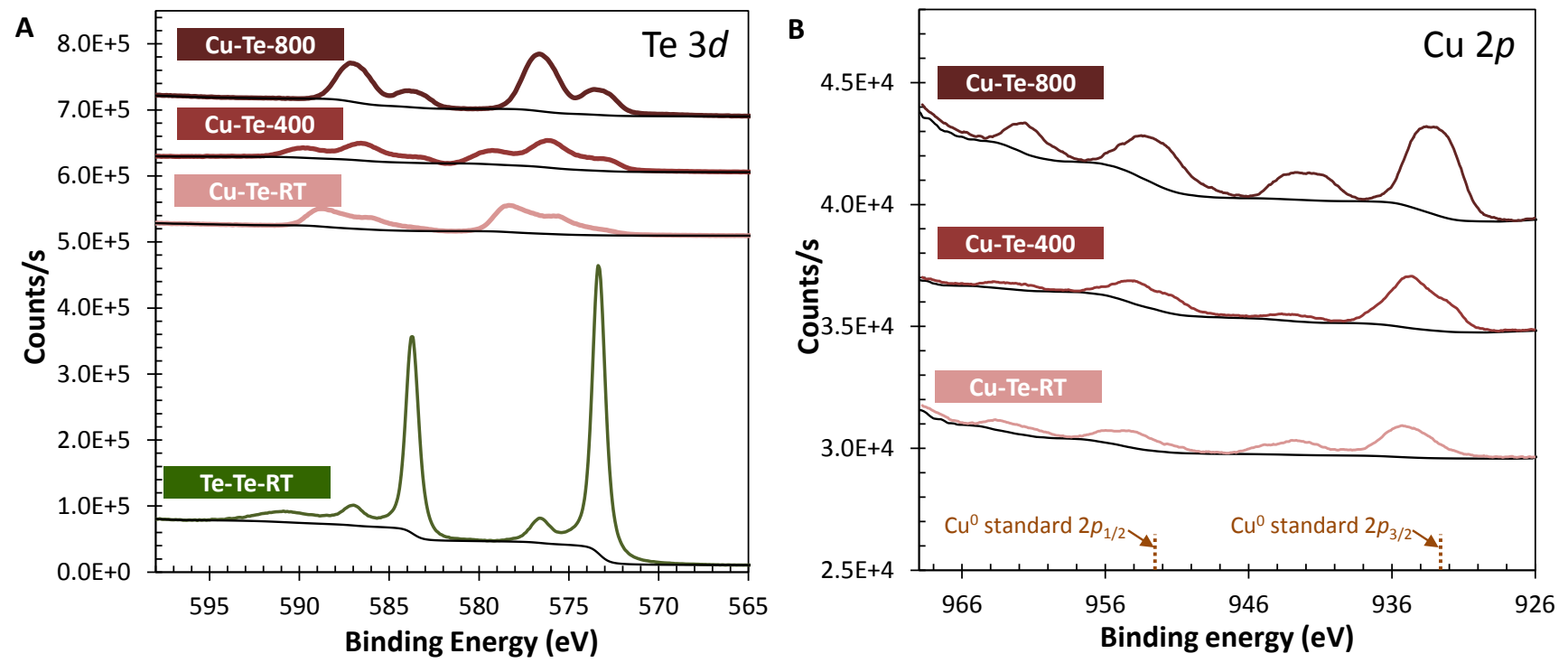

(A) Te $3 d$ and (B) $\mathrm{Cu} 2 p$ core XPS spectra of $\mathrm{Cu}$-Te particles from the flow heater at different operating temperatures; Te particles are included for comparison. 


\section{FIGURE S6}

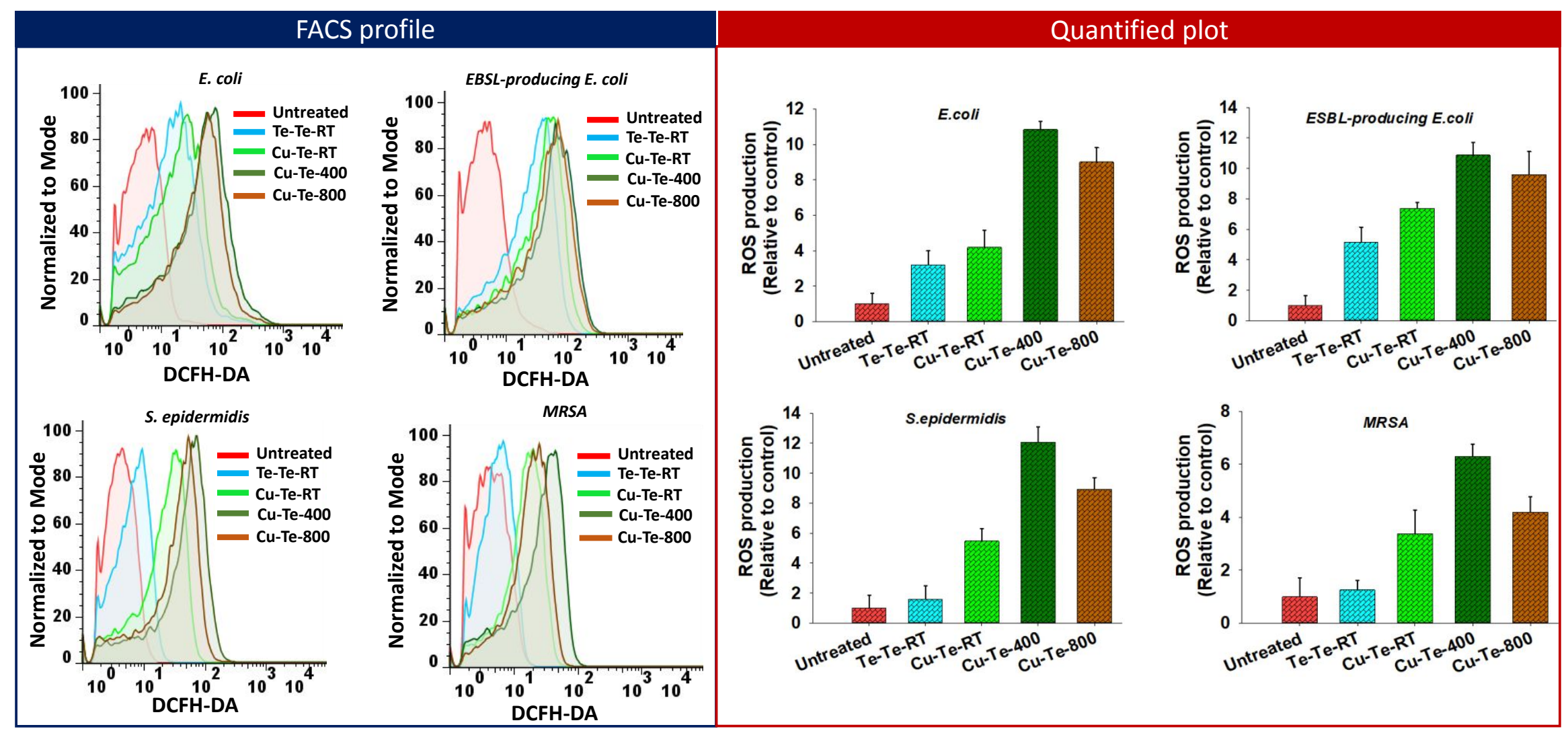

ROS production in $\mathrm{Cu}-\mathrm{Te}$ construct $(48 \mu \mathrm{g} / \mathrm{mL})$-treated $\left(24 \mathrm{~h}, 37^{\circ} \mathrm{C}\right)$ bacterial strains (E. coli, S. epidermidis, EBSL-producing E. coli, and MRSA). The generation was compared with that from individual Te NPs (at RT). 
FIGURE S7

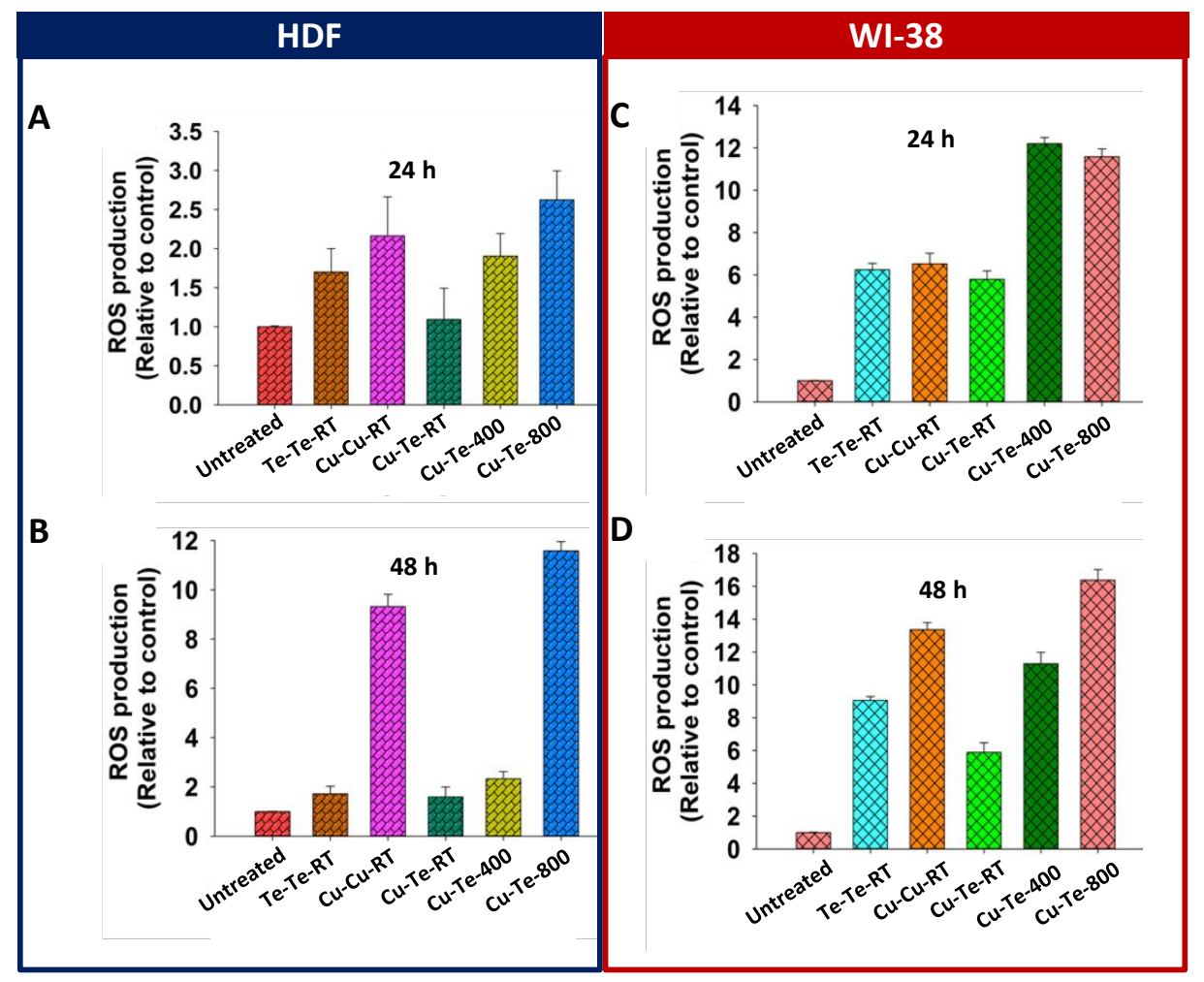

ROS production in (A, B) HDF and (C, D) WI-38 cells after 24- and 48-h treatments with $\mathrm{Cu}-\mathrm{Te}$ constructs $\left(50 \mu \mathrm{g} \mathrm{mL}^{-1} ; \mathrm{RT}, 400^{\circ} \mathrm{C}\right.$, and $\left.800^{\circ} \mathrm{C}\right)$; control experiments were performed with $\mathrm{Te}$ and $\mathrm{Cu}$ particles alone at RT. 


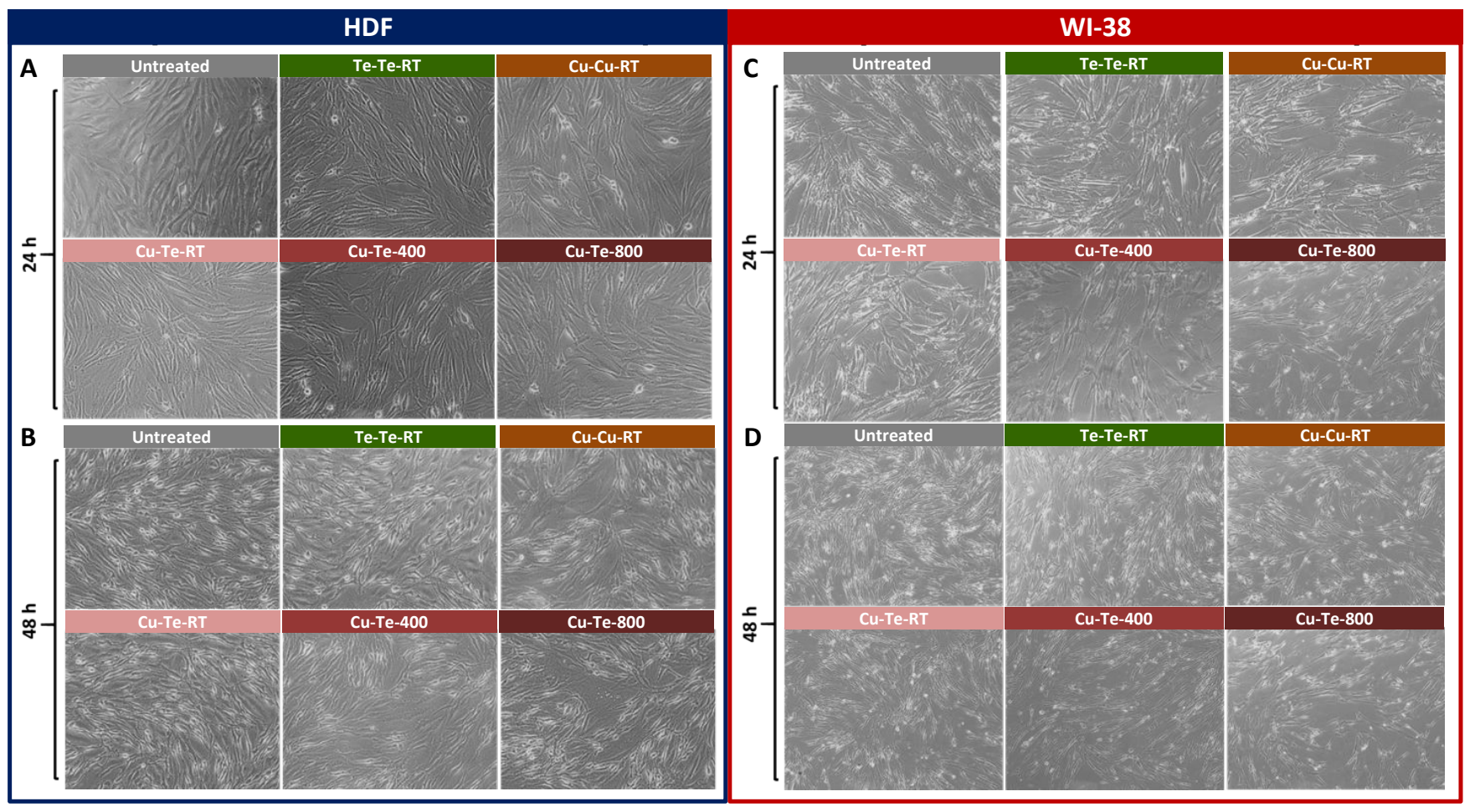

Optical microscope images of (A, B) HDF and (C, D) WI-38 cells treated with $\mathrm{Cu}-\mathrm{Te}$ constructs $(50 \mu \mathrm{g}$ $\mathrm{mL}^{-1}$; RT, $400^{\circ} \mathrm{C}$, and $800^{\circ} \mathrm{C}$ ); control cells were treated with Te and $\mathrm{Cu}$ particles (RT) for 24 and $48 \mathrm{~h}$. 
FIGURE S9

A

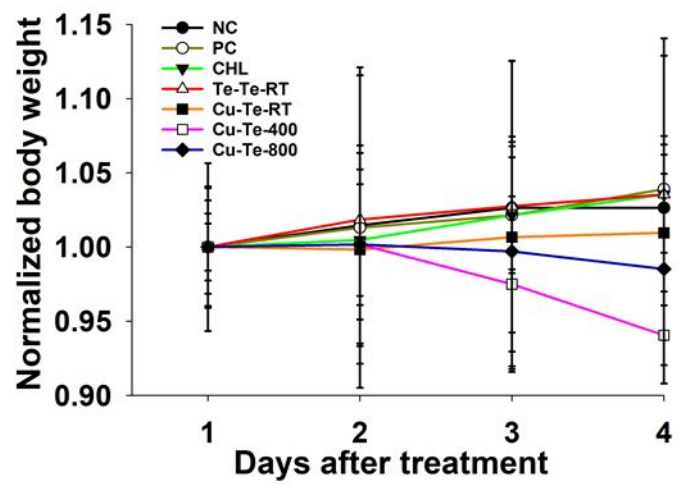

B

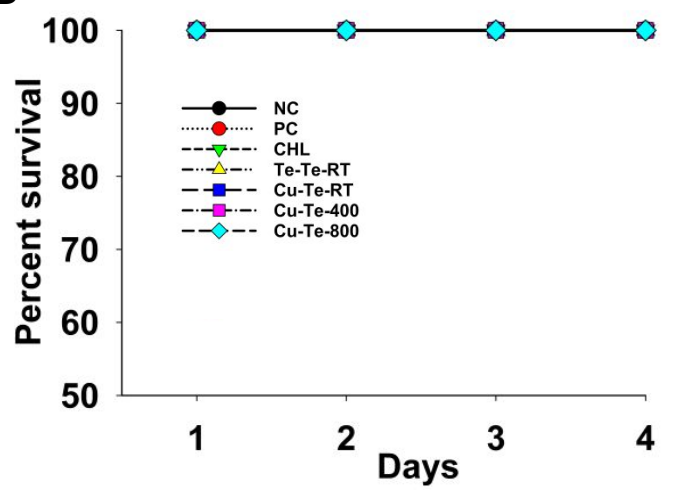

Monitored body weights (A) and survival rates (B) of $\mathrm{Cu}-\mathrm{Te}$ contrcu mice; NC, PC, and CHL were included for comparison. Monitored body weights (A) and survival rates (B) of the treated mice. 


\section{TABLE S1}

Summaries of histopathological-histomorphometric analyses of major organs of $\mathrm{Cu}$-Te-treated ICR mice

\begin{tabular}{|c|c|c|c|c|c|c|}
\hline Organs & Brain & Heart & Liver & Spleen & Lung & Kidney \\
\hline Groups & \multicolumn{6}{|c|}{ Abnormal finding } \\
\hline Untreated & $0 / 10(0 \%)$ & $0 / 10(0 \%)$ & $0 / 10(0 \%)$ & $0 / 10(0 \%)$ & $0 / 10(0 \%)$ & $0 / 10(0 \%)$ \\
\hline Te-Te-RT & $0 / 10(0 \%)$ & $0 / 10(0 \%)$ & $0 / 10(0 \%)$ & $0 / 10(0 \%)$ & $0 / 10(0 \%)$ & $0 / 10(0 \%)$ \\
\hline Cu-Te-RT & $0 / 10(0 \%)$ & $0 / 10(0 \%)$ & $0 / 10(0 \%)$ & $0 / 10(0 \%)$ & $0 / 10(0 \%)$ & $0 / 10(0 \%)$ \\
\hline Cu-Te-400 & $0 / 10(0 \%)$ & $0 / 10(0 \%)$ & $0 / 10(0 \%)$ & $0 / 10(0 \%)$ & $0 / 10(0 \%)$ & $0 / 10(0 \%)$ \\
\hline Cu-Te-800 & $0 / 10(0 \%)$ & $0 / 10(0 \%)$ & $0 / 10(0 \%)$ & $0 / 10(0 \%)$ & $0 / 10(0 \%)$ & $0 / 10(0 \%)$ \\
\hline
\end{tabular}

Values show fractions of abnormal fields/total observed fields.

G1, control; G2, Te-Te-RT-treated; G3, Cu-Te-RT-treated; G4, Cu-Te-400-treated; and G5, Cu-Te-800treated groups.

\section{TABLE S2}

Hematological analyses of ICR mice intraperitoneally treated with $\mathrm{Cu}-\mathrm{Te}$ constructs $\left(\mathrm{RT}, 400^{\circ} \mathrm{C}\right.$, and $\left.800^{\circ} \mathrm{C}\right)$, saline (Untreated), or individual Te particles (Te-Te-RT) for 18 days

\begin{tabular}{|c|c|c|c|c|c|}
\hline \multirow{2}{*}{ Indexes } & \multicolumn{5}{|c|}{ Treatment configuration (100 $\mathrm{mg} \mathrm{kg}^{-1}$ ) } \\
\hline & Untreated & Te-Te-RT & Cu-Te-RT & $\mathrm{Cu}-\mathrm{Te}-400$ & $\mathrm{Cu}-\mathrm{Te}-\mathbf{8 0 0}$ \\
\hline WBC $(k / \mu L)$ & $0.42 \pm 0.12$ & $0.53 \pm 0.17$ & $0.68 \pm 0.2$ & $0.63 \pm 0.36$ & $0.76 \pm 0.23$ \\
\hline$R B C(\mathrm{kM} / \mu \mathrm{L})$ & $5.18 \pm 0.27$ & $3.95 \pm 1.72$ & $5.50 \pm 0.34$ & $7.18 \pm 0.37$ & $7.28 \pm 0.67$ \\
\hline $\mathrm{HGB}(\mathrm{g} / \mathrm{dL})$ & $10.92 \pm 2.82$ & $10.40 \pm 2.97$ & $10.34 \pm 2.11$ & $13.08 \pm 0.59$ & $12.57 \pm 0.74$ \\
\hline HCT (\%) & $53.75 \pm 19.20$ & $26.80 \pm 11.03$ & $47.20 \pm 0.00$ & $67.40 \pm 2.93$ & $64.03 \pm 4.23$ \\
\hline MCV (fL) & $86.94 \pm 3.12$ & $88.80 \pm 1.55$ & $85.90 \pm 0.00$ & $93.87 \pm 1.95$ & $88.15 \pm 2.83$ \\
\hline $\mathrm{MCH}(\mathrm{pg})$ & $16.00 \pm 1.78$ & $24.13 \pm 24.84$ & $8.57 \pm 0.00$ & $18.22 \pm 0.63$ & $17.30 \pm 0.68$ \\
\hline MCHC (g/dL) & $18.49 \pm 1.96$ & $26.94 \pm 27.51$ & $9.98 \pm 0.00$ & $19.43 \pm 0.28$ & $19.65 \pm 0.26$ \\
\hline RDW (\%) & $15.75 \pm 1.21$ & $13.85 \pm 0.35$ & $13.60 \pm 0.00$ & $17.58 \pm 1.02$ & $16.90 \pm 0.82$ \\
\hline PLT $(k / \mu L)$ & $948 \pm 59$ & $988 \pm 22$ & $830 \pm 66$ & $1186 \pm 156$ & $1176 \pm 92$ \\
\hline MPV (fL) & $5.52 \pm 0.24$ & $5.34 \pm 0.41$ & $5.32 \pm 0.28$ & $6.47 \pm 0.53$ & $5.89 \pm 0.47$ \\
\hline PCT (\%) & $0.44 \pm 0.15$ & $0.16 \pm 0.12$ & $0.38 \pm 0.15$ & $0.76 \pm 0.06$ & $0.69 \pm 0.10$ \\
\hline PDW 10 (GSD) & $17.22 \pm 0.86$ & $16.5 \pm 0.45$ & $16.12 \pm 0.42$ & $17.12 \pm 0.45$ & $17.22 \pm 0.25$ \\
\hline
\end{tabular}

$\mathrm{MCV}$, mean cell volume; $\mathrm{MCH}$, mean corpuscular hemoglobin; $\mathrm{MCHC}$, mean corpuscular hemoglobin concentration; RDW, red cell distribution width; MPV, mean platelet volume; PCT, plateletcrit; and PDW, platelet distribution width. 


\section{TABLE S3}

Summaries of biochemical analyses for blood serum in ICR mice intraperitoneally treated with $\mathrm{Cu}-\mathrm{Te}$ constructs $\left(\mathrm{RT}, 400^{\circ} \mathrm{C}\right.$, and $800^{\circ} \mathrm{C}$ ), saline (Untreated), or individual Te particles (Te-Te-RT) for 18 days

\begin{tabular}{|c|c|c|c|c|c|}
\hline \multirow{2}{*}{ Indexes } & \multicolumn{5}{|c|}{ Treatment configuration (100 mg kg-1) } \\
\hline & Untreated & Te-Te-RT & Cu-Te-RT & Cu-Te-400 & $\mathrm{Cu}-\mathrm{Te}-800$ \\
\hline $\begin{array}{l}\text { ALP-PS } \\
(U / I)\end{array}$ & $269.5 \pm 49.8$ & $263.0 \pm 49.3$ & $277.2 \pm 48.0$ & $209.5 \pm 74.3$ & $265.0 \pm 54.3$ \\
\hline $\begin{array}{l}\text { GOT-PS (AST) } \\
(\mathrm{U} / \mathrm{I})\end{array}$ & $53.7 \pm 10.19$ & $35.4 \pm 5.31$ & $34.2 \pm 4.43$ & $65.0 \pm 12.75$ & $41.5 \pm 6.45$ \\
\hline $\begin{array}{l}\text { GPT-PS (ALT) } \\
(\mathrm{U} / \mathrm{I})\end{array}$ & $54.5 \pm 22.75$ & $30.4 \pm 5.85$ & $28.6 \pm 3.5$ & $72.8 \pm 33.94$ & $35.5 \pm 14.01$ \\
\hline $\begin{array}{l}\text { TP-PS } \\
(\mathrm{g} / \mathrm{dL}) \\
\end{array}$ & $4.89 \pm 0.25$ & $4.74 \pm 0.15$ & $4.72 \pm 0.16$ & $5.17 \pm 0.22$ & $4.87 \pm 0.25$ \\
\hline $\begin{array}{l}\text { ALB-PS } \\
(\mathrm{g} / \mathrm{dL})\end{array}$ & $2.36 \pm 0.14$ & $2.18 \pm 0.14$ & $2.22 \pm 0.08$ & $2.65 \pm 0.17$ & $2.3 \pm 0.14$ \\
\hline $\begin{array}{l}\text { BUN-PS } \\
(\mathrm{mg} / \mathrm{dL})\end{array}$ & $22.2 \pm 1.56$ & $21.0 \pm 3.22$ & $23.0 \pm 0.88$ & $21.4 \pm 2.93$ & $17.8 \pm 4.77$ \\
\hline $\begin{array}{l}\text { CRE-PS } \\
(\mathrm{mg} / \mathrm{dL})\end{array}$ & $0.12 \pm 0.02$ & $0.10 \pm 0.00$ & $0.12 \pm 0.04$ & $0.28 \pm 0.09$ & $0.13 \pm 0.05$ \\
\hline $\begin{array}{l}\text { GLOB } \\
(\mathrm{g} / \mathrm{dL})\end{array}$ & $2.52 \pm 0.12$ & $2.56 \pm 0.05$ & $2.50 \pm 0.10$ & $2.53 \pm 0.12$ & $2.58 \pm 0.12$ \\
\hline ALB/GLOB & $0.95 \pm 0.04$ & $0.86 \pm 0.05$ & $0.88 \pm 0.04$ & $1.05 \pm 0.10$ & $0.90 \pm 0.00$ \\
\hline BUN/CRE & $128.5 \pm 0.0$ & $112.15 \pm 4.0$ & $110.0 \pm 0.0$ & $83.8 \pm 24.02$ & $121.5 \pm 0.0$ \\
\hline
\end{tabular}

